\title{
The spread characteristics of music videos relating to COVID- 19 in China online video platforms
}

\author{
Jiangnan Zhao ${ }^{\mathrm{a}, 1, *}$, Cheng Chen ${ }^{\mathrm{b}, 2}$, Lai Zhao ${ }^{\mathrm{c}, 3}$ \\ ${ }^{a}$ Nanjing Normal University, No. 1 Wenyuan Road, Nanjing 210023, China \\ b Nanjing Normal University, No. 1 Wenyuan Road, Nanjing 210023, China \\ c Xinjiang Agricultural University, No. 311 Nongda East Road, Shayibak District, Urumqi City, Xinjiang Uygur Autonomous Region, 830052, China

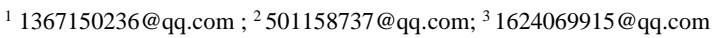 \\ * corresponding author
}

ARTICLE INFO

Article history

Received 2020-04-14

Revised 2020-04-22

Accepted 2020-06-03

Keywords

COVID-19

Music

Video

Platform

Characteristics

\begin{abstract}
This paper aims to explaining how was the behavior of music videos in China and online video platforms during the outbreak of COVID-19, which is related to the situation of COVID-19, were taken as the research object. Firstly, the data were collected and proceeded, then the time distribution, the types of the author, geographical distribution, theme, the types of music were gathered for data statistics. Secondly, this study analyzed the characteristics of the production and spread of these music videos, which were displayed visually. This research is library research. The author conducted in-depth studies related to various literacies that explain the objects and subjects of this study. Finally, this paper systematically analyzed the effect and influence of social literature and art in a public event.
\end{abstract}

This is an open access article under the CC-BY-SA license.

\section{Introduction}

COVID-19, which broke out in late 2019, is the most widespread public health emergency in the history of new China. The outbreak is the command for us; prevention and control is the responsibility for people. Under the strong leadership of the central committee of the Chinese communist party, people of all ethnic groups in China, on all fronts and in all fields, have made full use of their advantages to spread positive energy for COVID-19's prevention and control, which contribute to overcoming the people's war for the prevention and control of COVID-19, the endless war and the defensive war with more substantial confidence and stronger will [1]. President Xi Jinping has pointed out that literature and art should be "people-centered," which emphasizes that artistic creation and media working should listen to the voice of the audience, pay attention to social benefits and meet the needs of the overwhelming majority of the people [2].

In the process of epidemic prevention and control, traditional media, network media, new media, web media, individuals, etc., they quickly made and published a large number of literary and artistic works enhancing national cohesion via using a variety of art forms. In the meanwhile, these works produced extensive and positive social influence as well as playing a pivotal role to mobilize the whole social strength [3]. In the practice of social media spread at this stage, music video ("MV" for short), as an essential art form with a widespread, has significant social effects, which is closely related to the artistic quality, production characteristics and characteristics of spread of MV [4]. MV is an art form constituted by the cooperation of music and pictures. The perfect combination of sound and image can significantly enhance the atmosphere and arouse emotion, so that makes the audience immersed in the atmosphere created by it [5]. 
The time length of the video form of MV is generally about 3 minutes, which conforms to the characteristics and needs of video spread in the era of mobile Internet, and provides a realistic basis for a more efficient and wide range. At the same time, the advantages of MV, such as low production cost, short production time, and small access threshold, objectively contribute to the extensive spread and profound impact of MV in this outbreak [6]. From the perspective of demand, the mass of useful information conveyed by MV accurately responds to the information needs of the public promptly and reasonably and appropriately defuses diverse and complicated social emotions. It not only provides feedback for the information needs of the society but also injects energy into the functions of public management and service departments [7].

Therefore, this study took MV relating to COVID-19 in China online video platforms during the outbreak of COVID-19 as the research object, systematically analyzed its characteristics of spread and rules, and provided an experience for future spread practice.

\section{Theoretical Framework}

The diversity of types of musical works and music publication media that grow in people's lives have different positions and functions. Their music is used as a medium of expression to realize artistic works called compositions, media for educational activities both in schools and non-school education, used as a medium of communication between ethnic groups and between countries. The variety of functions of regional music in every aspect of the social life of a nation [3].

Music in society has various functions, including functions that are individual and social. Tasks that are individual, namely as an expression or expression of the soul and as inner satisfaction for its creator. The social function of music has a significant role in various fields of community life, including being a medium of entertainment, communication, education, commerce, military, and religion [8].

Speaking of music is talking about a symbol of things related to the ideas and behavior of a society. Music is part of the arts; art is one element of culture and is one of the universal human needs that is never separated from society. Music is one of learning, meaning music was created by humans to meet their needs for beauty. It can be interpreted that music has a function in human life.

The digital age makes human life more comfortable in all aspects. The term digital is often referred to as the emergence of the internet network or information and communication technology. This ease has an impact on the development of the music industry. Marketing music releases in the digital era are now not only about album production or Extended Play (EP), which is blessed on solid discs, cassette tapes, or compact discs, which are then distributed to music stores in each city or region [4].

Musicians can now efficiently distribute their work through various online media, both through social media, streaming services, and web profiles. The emergence of social networking sites like My Space in the early 2000s is one marker that the digital era has penetrated the music ecosystem. My Space provides Space for everyone to create pages with their profiles, including their music. Continuing in 2006, Reverbnation specifically offered a meeting place for musicians who wanted to release their work with listeners. Through Reverbnation, musicians who have just released their work will have the opportunity to be heard more widely and have the chance to play in the festival without going through label intermediaries [9].

The digital age makes boundaries between countries or territories increasingly blurred; this also applies to the distribution of music releases. Through digital media, musicians can freely distribute their work, compete with millions of jobs of music throughout the world, and make the exclusion of exclusion from musicians who first entered the world of music. Now, listeners can be freer to look for works of music that truly fit their musical tastes [10].

Among the conveniences of the digital age in terms of the distribution of music releases, it seems to note the difficulties that musicians will encounter. One of them, the competition between works of musicians which at first was limited to a few limitations, now everything has become more open and free. Those who are said to be successful in general are those who have succeeded in attracting as many listeners as possible. 
Of course, facing this difficulty requires some adjustments and strategies for musicians who want to distribute their work through digital media. Here are some of the strategies needed to distribute music releases in the digital age [9]:

i. Substance over form, which means giving importance to the content on the way and packaging. The most crucial thing in a musical work is the quality of the music itself, not just the packaging or how intense the music is being marketed. Some actions that are deemed not sufficiently "mature" in an instant can indeed attract the enthusiasm of the listeners, but do not last long. Therefore, to get long-lasting, more organic and real passion, the quality of the music is more important. Unbelievably attractive marketing will not continue to manifest enthusiasm if indeed the work is deemed to be of poor quality.

ii. Digital platform. Use digital platforms well. Now there are lots of platform choices to distribute music releases. Starting with the streaming platform, various options are available, including Spotify, iTunes, Deezer, JOOX, Youtube Music, Soundcloud, etc. On the videosharing platform, Youtube is available. Then, on the web profile platform Instagram, Twitter, Facebook, Bandcamp, Reverbnation, etc. are also possible.

iii. The availability of various marketing media requires musicians to be more observant and wise in using it. Musicians can choose a platform according to their promotional needs and the type of work they will distribute. Through a streaming platform, musicians can distribute their work through sound media. As for the video-sharing platform, musicians can distribute neatly packaged forms of work in the way of videos, such as video clips, live performance videos, video rehearsals, and videos in the arrangement process. Through the web profile platform, the musicians can provide more personal information about the musician's profile, including updating the latest news, building and strengthening the branding and image and persona of the musician, as well as maintaining interaction with his listeners.

iv. Maintain engagement through social media. After getting attention from the listeners, in general, the general behavior of the listeners is to find a medium of continuous interaction with the musician. In the digital age, social media is the right and most common place to use it, namely Instagram, Twitter, Facebook, and others. Musicians can maintain engagement with their listeners in several ways, including by continuing to provide daily updates on the activities of musicians, updating the latest information about the progress of the work, live performance, rehearsal, and of course the question and answer feature helps musicians to read and respond directly message from the listeners.

v. Little Spam. Before the ease brought about by the digital era, musicians typically made a form of a collection of music that was then poured into an album or EP. But now, they are more facilitated because they can distribute their work one by one (little spam) because it is not bound by an obligation to make a collection of works first. This requirement is undoubtedly related to the production costs that must be spent by the label to promote a musician, as an example of this experience experienced by the Band Rotors at the beginning of its appearance. The shelter label required him to prepare ten songs as a condition of entering the recording kitchen, but at that time, they only had three songs. Finally, within the specified deadline, the Band Rotor must meet that target. Of course, this will no longer be experienced by musicians who will distribute their work through digital media.

vi. Merchandise cannot be separated from a band/musician and becomes a separate identity for the band or musician. Merchandise can be in the form of t-shirts, hats, bags, and so on. The proverb says, one way to support musicians for listeners in addition to watching their shows is by buying their merchandise. With quality merchandise, musicians not only receive material profits from the sale of merchandise but also benefit indirectly from listeners who use their merchandise. In the end, making a piece of music known and widespread to listeners is not the primary mission in creating a work. A work that is produced honestly and full of messages will find its gap to reach the listeners. Being a musician does not merely make our music work famous but also relates to the news that we carry. With the ease of digital media as a means of promoting musical works, it does not then become an omission for musicians to create works of not quality just for the sake of seeking fame. 


\section{Method}

One type of research, when viewed from the place of data collection, is library research-called library research because of the data or materials needed to complete the research originating from the library in the form of books, encyclopedias, dictionaries, journals, documents, magazines and so on. To facilitate the study of literature, indeed, a researcher is required to know and understand the organization and work procedures library. This is important to more easily obtain and access materials or resources needed [11].

So important is the study of literature, that it is impossible for a review to be carried out without first doing it, moreover in library research must read many reports relating to the focus of the study. More than fifty percent of the activities in the entire research process is reading, and because of this, the reading source is an essential part of research support [12].

In finding reading sources, a researcher must be selective because not all can be used as data sources. It is necessary to avoid the use of older reading sources and the choice of more up-to-date sources. Early origins may contain theories or concepts that are no longer valid because the truth has been refuted by newer arguments or the results of later research. Therefore, to get data or materials from books and other literature that meet the two principles above requires foresight, perseverance, and craftsmanship in finding data, both primary and secondary data sources [13].

The Report of China's Business Situation in Online Video in 2018 pointed out," After years of development, China's online video platform companies already have iQiyi, Tencent Video, Youku, Sohu Video, Phoenix Video, Mango TV, PPTV, etc., which have become the backbone of China's online video industry" [14]. In this study, five representative portal websites, including iQIYI, Tencent Video, Youku, Sohu Video, and Phoenix Video, were selected as data source platforms. At the same time, Bilibili, a current popular live video platform, is included in the data source platform. The data source platform for this study is the above six video websites. The time range of this study is from January 23rd, 2020, to February 22nd, 2020.

In consequence, a search was performed on six video platforms with "the outbreak of COVID$19, \mathrm{MV}^{\prime}$ as the keyword, and a total of 3,039 results were obtained within the time range of this study. After manual screening steps such as merging the same videos released on multiple platforms and removing inconsistent types of videos, 379 valid MVs were obtained as the final research object. In order to carry out a comprehensive analysis of multi-feature elements, this study took 379 MVs as the research object to perform statistical processing on the basic data of the spread elements. The process is as follows:

a) Sort out the MV release date and make statistics.

b) Sort out author types of MVs and perform classification statistics. (3) Sort out the release area of MV and make statistics with provincial units.

c) Statistical analysis of word frequency for 379 MVs titles.

d) Count the number of original music and unique music forms.

\section{Results and Discussion}

The epidemic situation is closely related to everyone. With the aggravation of the condition and the upgrade of prevention and control measures, the attention of the public has further increased, and these changes can also be reflected in the distribution of MV release time [15]. Sorting out the release date of $379 \mathrm{MVs}$, the results are shown in Fig .1. It can be seen that although the inside of the graph shows a tortuous situation, the overall trend is an upward trend, which is highly consistent with the gradual increase in social attention. Specifically, the first relevant MV appeared on January 27 th, and then steadily increased. The first peak appeared on February 1st; this stage is the outbreak period of the release number [16]. After a long period of time, the number of releases showed high fluctuations and an overall upward trend. There are Seven peaks, including February 3rd (16), February 6th (20), February 8th (20), February 11th (17), February 14th (26), February 17th (19), and February 22nd (31). This trend is expected to continue for some time. This stage is the continuous growth period in the number of releases. As the epidemic condition is further effectively 
controlled and ended, it is expected that the number of MV releases will show a fluctuant downward trend, this stage is a period of recovery.

Fig.1.

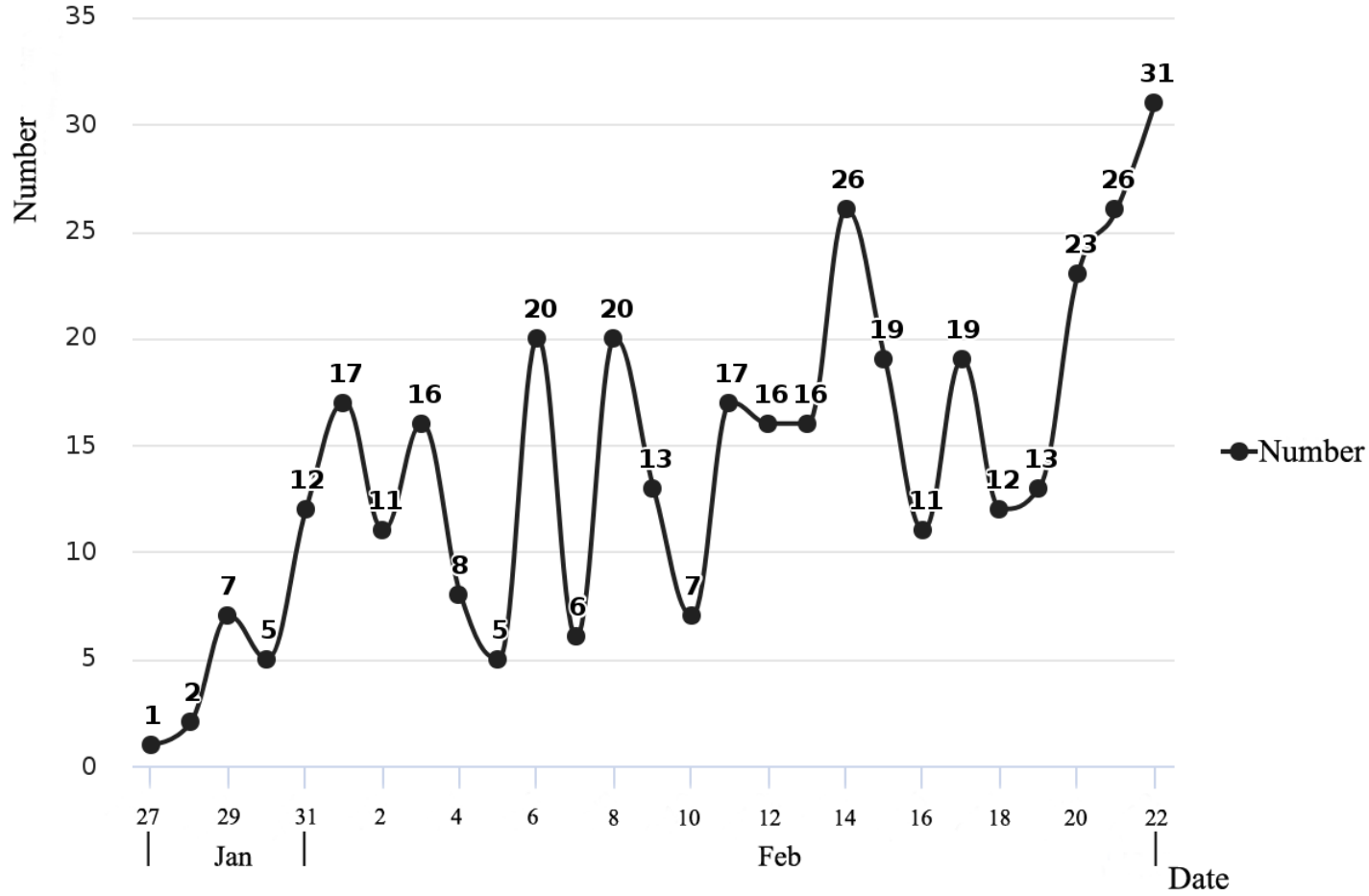

Fig. 1.The distribution of MV release time.

Creators of $379 \mathrm{MVs}$, including party and government organs, public institutions, military, enterprises, and individuals. According to the characteristics of the attributes, this study classifies the party and government organs and public institutions as public administration and public service departments. Hence, there are four types of authors: "Public Administration and Public Service Department, Military, Enterprises, and Individuals." Fig. 2 shows the statistics of the authors of 379 MVs. Among them, public administration and public service departments account for the most significant proportion, reaching 54\%, followed by individuals accounting for $32 \%$, enterprises accounting for $12 \%$, and militaries accounting for $2 \%$. It can be seen that in public events, the official open spread agencies, including the public administration and public service departments and the military, are still strong and mainstream, and are still the main body that bears the responsibility for public opinion [17]. Simultaneously, the rapid development of web media represented by companies and individuals has actively used artistic methods to express ideas and express emotions in public events, gradually changing from the previous "receiving signal" to the current "transmitting signal" and becoming an essential part of public opinion responsibility [17].

For a long time, the official open spread agencies have a large volume, extensive influence, and high authority. This is highly consistent with the performance of the public administration and public service departments and the military in this study. As Marshall McLuhan wrote it, "The medium is the message" [18]. In the era of new media, official public spread agencies must also actively adapt to the tide of the times, to follow the trend and take advantage of the pattern, and actively transform to make government public spread more diverse [19]. A flexible and more acceptable way of expression, including MV and other arts, which is necessary to conduct public opinion and emotion. Therefore, it can achieve the purpose of general management and service.

The development of we media has profoundly changed the media landscape [20]. Web media constituent units represented by enterprises and individuals are increasingly producing and communicating cultural works such as MV on the online platforms, expressing opinions, and participating in interactions, which makes the content form diversified and the timeliness of 
information dissemination greatly enhanced. In other words, it provides a revolutionary filling for public governance in this open event [21].

Fig.2.

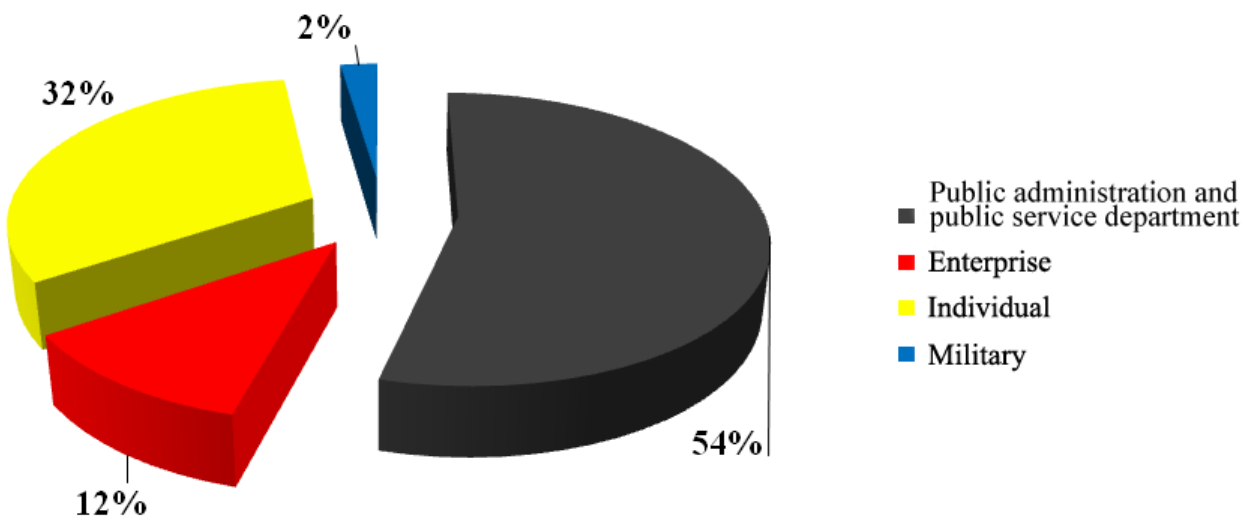

Fig. 2. The distribution of MV author types

According to the display of geographical spatial distribution, differences in media responsiveness, and MV production capabilities of each provincial-level administrative units can be analyzed. In this study, using ArcGIS spatial statistical methods, the distribution areas of MVs were sorted out using the provincial-level administrative units. As shown in Fig.3, the depth of the color represents the number of releases. On the whole, all provincial-level administrative units have related MV released and disseminated. Among them, the central and eastern regions are darker in color, and the western and northeastern areas are relatively lighter, which coincides with the "HeiheTengchong Line" [22]. To be specific, four provincial-level administrative units, including Beijing, Shandong, Guangdong, and Henan, have the darkest color. Hence, the number of releases is more than in other provinces. This is highly related to political, economic, cultural, demographic, and other macro factors that affect the creative ability and media market environment. Also, the prevention and control methods and efforts of Henan province in this outbreak are relative "hardcore", which has been listed on the popular search list of Sina Weibo for many times. In the meanwhile, it is mutually confirmed by the performance of the spatial distribution of media spreadability [23].

At the same time, Russia, America, Italy, Malaysia, Thailand, and other countries have a certain amount of relevant MV that posted on China's online video platforms, which is related to the development trend of international integration of network media. 


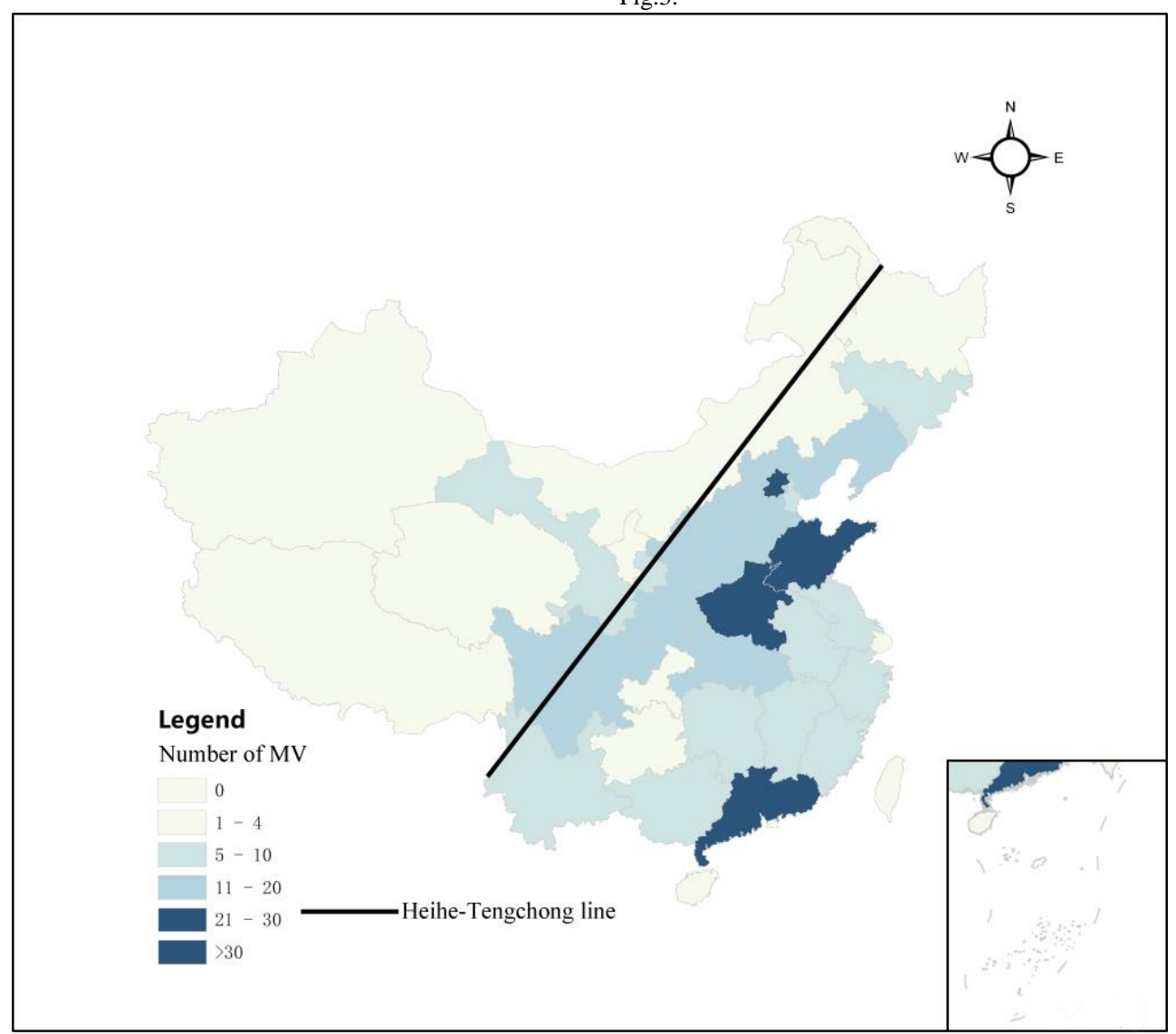

Fig. 3. The display of the geographical spatial distribution of MV.

TF-IDF is a text weighting technique that is commonly used, where TF (Term Frequency) is the word frequency, and IDF (Inverse Document Frequency) is the inverse text frequency index [24]. The TF-IDF algorithm plays a vital role in evaluating the importance of a word to a file set or a file in a corpus [25]. Table 1 shows that the TF-IDF algorithm, In this study, is used to perform statistical processing on the titles of $379 \mathrm{MVs}$, and the top 50 keywords were obtained. Fig. 4 shows the tag cloud based on keywords appearing in Table 1.

Table 1. The display of word frequency

\begin{tabular}{|c|c|c|c|c|c|c|c|}
\hline $\begin{array}{c}\text { IDF } \\
\text { ranking }\end{array}$ & words & $\begin{array}{l}\text { part of } \\
\text { speech }\end{array}$ & frequency & IDF ranking & words & $\begin{array}{l}\text { part of } \\
\text { speech }\end{array}$ & frequency \\
\hline 1 & Outbreak & Noun & 18 & 26 & Light & Noun & 3 \\
\hline 2 & Retrograde & Verb & 15 & 27 & Back shadow & Noun & 3 \\
\hline 3 & Most beautiful & Adjective & 21 & 28 & Hello & Noun & 3 \\
\hline 4 & We & Pronoun & 21 & 29 & Warm & Verb & 3 \\
\hline 5 & Come on & Verb & 15 & 30 & White & Noun & 3 \\
\hline 6 & Come together & Idiom & 6 & 31 & Come back & Verb & 3 \\
\hline 7 & Angel & Noun & 10 & 32 & Strength & Noun & 3 \\
\hline 8 & Hero & Noun & 9 & 33 & Hold up & Verb & 2 \\
\hline 9 & Hope & Verb & 8 & 34 & Certainly & Adverb & 3 \\
\hline 10 & Life & Noun & 7 & 35 & prevent & Verb & 2 \\
\hline 11 & Guard & Verb & 6 & 36 & Depart & Verb & 2 \\
\hline 12 & Spring & Time word & 6 & 37 & Make a living & Verb & 2 \\
\hline
\end{tabular}




\begin{tabular}{|r|c|c|c|c|c|c|c|}
\hline 13 & Trust & Verb & 5 & 38 & Unite & Verb \\
\hline 14 & Believe & Verb & 5 & 39 & Stick to & Verb \\
\hline 15 & Answer & Noun & 5 & 40 & Join & Verb \\
\hline 16 & Fight & Verb & 3 & 41 & Forerunner & Noun \\
\hline 17 & Mask & Noun & 3 & 42 & Bloom & Verb \\
\hline 18 & Wings & Noun & 4 & 43 & Warrior in white & Noun \\
\hline 19 & Tomorrow & Time word & 4 & & 44 & Motherland & Noun \\
\hline 20 & Apply for battle & Verb & 2 & & 45 & Chinese love & Noun \\
\hline 21 & Undertake & Verb & 3 & & 46 & This time & Pronoun \\
\hline 22 & Combat & Noun & 3 & & 47 & Dedicate & Verb \\
\hline 23 & Connect & Verb & 3 & & 48 & Figure & Noun \\
\hline 24 & Heart to heart & Noun & 2 & & 49 & All people are soldiers & Idiom \\
\hline 25 & Mission & Noun & 3 & & 50 & Courage & Noun \\
\hline
\end{tabular}

From the ranking point of view, keywords such as "retrograde, most beautiful, we, come on, come together, angel, hero, hope and life" are at the top of the list. They are the primary creative theme of the relevant MV. Generally, They express the acclaim of the staff working against the epidemic and the determination to win the battle against the virus. Scholar Zhao Bo divides the acceptance psychology of MV audience into emotion demand psychology, idol worship psychology, entertainment pastime psychology, learning imitation psychology, and group psychology [26]. In this outbreak, the acceptance psychology of the MV audience is mainly based on emotional needs. These spread themes are consistent with the psychological and emotional expectations of the public in the outbreak. From the part-of-speech point of view, the main things are nouns and verbs in which the pronoun "we," the adjective " most beautiful," idiom, "come together," and the time word "spring" is also used very frequently.

Fig.4.

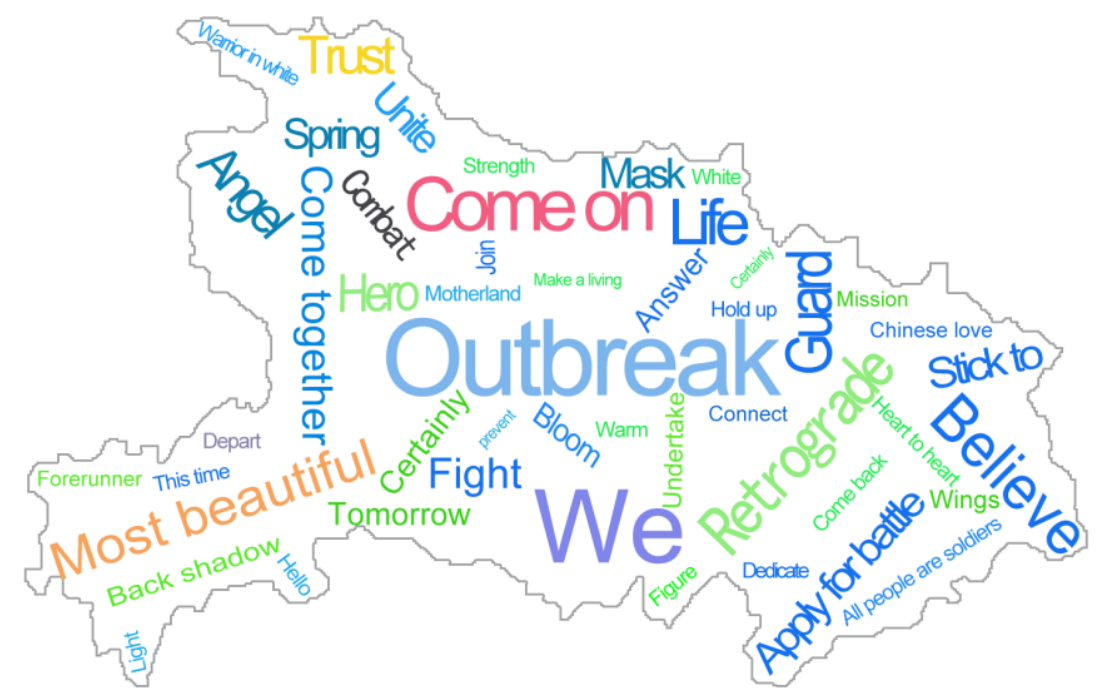

Fig. 4. The tag cloud of the Word frequency

MV tells a story with images and conveys warmth and strength with music. The spread of music has a close relationship with society [27]. In this outbreak, the range of MV has significantly benefited from the assistance of music. In this study, the statistics of music originality and music 
form of 379 MVs are summarized. Fig. 5 shows the proportion of original music. In the $379 \mathrm{MVs}$, it reached $94 \%$ and the non-original $6 \%$. Owing to original music can be more recognized in terms of production level and public opinion effect so that the higher the degree of attention received, the better the impact in the spread processing [28]. In terms of musical form, in addition to the standard forms such as Chinese pop music, there are various musical forms including rap, English songs, children's songs, Peking opera, Allegro, Jin opera [29], Chinese dialects, Shaanxi Opera [30], Thai songs, Russian songs, etc., and Have a particular influence on the spread.

Mozart said, "The misery of life will not overwhelm me. The joy in my heart is not my own. I put joy into the music to make the world feel happy." These music videos born in this outbreak will deliver hope, courage, and motivation, and all people and things will be remembered.

Fig.5.

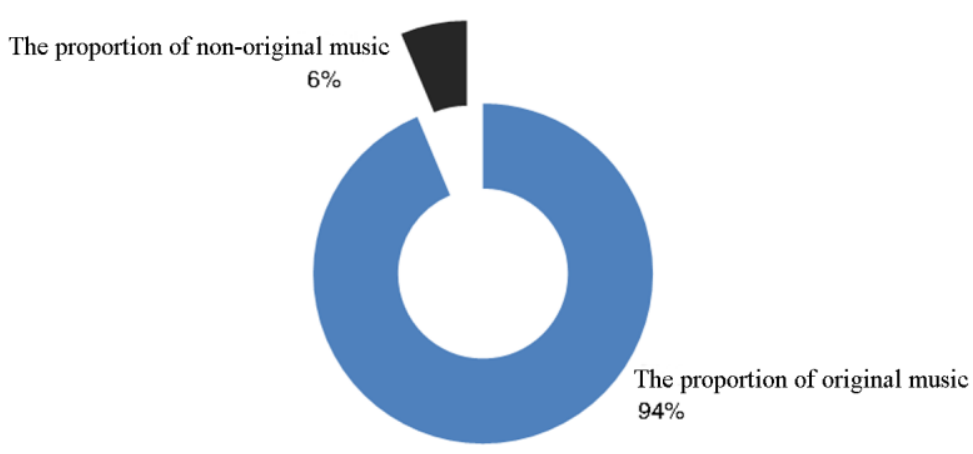

Fig. 5.The proportion of original and non-original music.

\section{Conclusion}

Through the statistical analysis of the release time, author type, geographical distribution, theme word frequency, music, and other aspects of $379 \mathrm{MVs}$, the following conclusions are mainly obtained. First, in terms of time distribution, the spread of related MV is divided into three stages: the outbreak period, the continuous growth period, and the recovery period. Second, in terms of the types of authors, the official public spread agency is the main body that takes responsibility for public opinion. Meanwhile, we media represented by enterprises and individuals has become an essential part of undertaking the obligation of public opinion. Third, in terms of geographical spatial distribution, the number of central and eastern regions is significantly higher than that of western and northeastern areas, whose boundary line coincides with the "Heihe-Tengchong Line. " Fourth, in terms of keywords frequency, keywords such as "retrograde, most beautiful, us, come on, come together, angel, hero, hope, life," proceeding from the emotional needs of the general public, whose TF-IDF ranking is higher than others. Fifth, in terms of music distribution, the main things are original music and Chinese popular music, while including rap, English songs, Peking opera, Allegro, and other forms of music. 


\section{References}

[1] Y. Z. Y, "Information presentation and media performance in COVID-19 's report," The Press, pp. 12$15,2020$.

[2] H. Ning, "Xi Jinping's 'people-centered' view of literature and art,"" Chinese Lang. Lit. Res., pp. 11-15, 2019.

[3] F. F. Lotan, "Making a positive internet through Socmed Agawe Guyub," Int. J. Commun. Soc., vol. 1, no. 1, pp. 9-16, 2019, doi: 10.31763/ijcs.v1i1.22.

[4] U. Jandevi, “Communication strategy to improve women's political participation in Indonesia," Int. J. Commun. Soc., vol. 1, no. 2, pp. 68-81, Sep. 2019, doi: 10.31763/ijcs.v1i2.46.

[5] Yunhe, "On the theme expression of MV Image Music," Media Forum, vol. 2, p. 169, 2019.

[6] A. Meng, "The concept definition, classification and five development stages of MV," Music Commun., pp. 72-82, 2013.

[7] M. Hong, "Research on the public relations strategy of emergency government news communication," $J$. Chang. Educ. Inst., vol. 29, pp. 44-45, 2013.

[8] D. Fadillah, "Belt Road Initiative in the Simulacra Analysis (Indonesia - China Relationship in the Perspective of Indonesian Students in Nanjing City)," ASPIKOM, vol. 5, no. 1, pp. 63-71, 2020.

[9] M. Kartomi, "The Saman Gayo Lues Sitting Song-Dance and its Recognition as an Item of Intangible Cultural Heritage," Yearb. Tradit. Music, 2013.

[10] Rob Cover, "Interactivity, Digital Media, and the Text," in Digital Identities, Academic Press, 2016, pp. 71-101.

[11] S. J. Tracy, "Qualitative quality: Eight a"big-tent" criteria for excellent qualitative research," Qual. Inq., 2010, doi: 10.1177/1077800410383121.

[12]A. Ghofar, U. Abubakar, and M. Azhar, "TAZKIYATUN NAFS AS A STRENGTH BASE OF TEACHER PERSONALITY COMPETENCY," IJISH (International J. Islam. Stud. Humanit., vol. 1, no. 2, p. 128, Feb. 2019, doi: 10.26555/ijish.v1i2.559.

[13]C. C. and G. Symon, Qualitative Methods in Organizational Research. London: Sage Publication, 1994.

[14] iResearch Consulting, "The Report of China's Business Situation in Online Video," iResearch Consulting, 2018. [Online]. Available: https://www.iresearch.com.cn/Detail/report?id=3216\&isfree=0.

[15]J. Ping, "The dissemination of epidemic information should be led by the needs of the audience: Observation of domestic Public opinion Field after COVID-19 's outbreak," The Press, pp. 6-8, 2020.

[16]J. Xia, "Research on the role of news communication in government crisis management," Public Commun. Sci. Technol., vol. 7, pp. 11-20, 2015.

[17] Y. Bing, "An analysis of the influence of we media communication on traditional news communication," Journal. Commun., pp. 33-35, 2018.

[18] M. McLuhan, Understanding Media: The Extensions of Man. Massachusetts: MIT Press, 1994.

[19] T. Fang, "The research on the government public communication in the new media environment," Hunan, 2010.

[20]D. Junchuang, "The function and influence of the Application of we media in Public Management," West China Broadcast. TV, vol. 2021, 2020.

[21] W. Guangzhao, "Individual Media: a New form of we Media Communication Mode," Media Obs., pp. 67-71, 2019.

[22]D. Fadillah, L. Zhenglin, and D. Hao, "Big Data and the Revolution of Political Campaign in Indonesia," in Proceedings of the 2019 Ahmad Dahlan International Conference Series on Education \& Learning, Social Science \& Humanities (ADICS-ELSSH 2019), 2019, doi: 10.2991/adics-elssh-19.2019.19.

[23]"The prevention and control methods and efforts of Henan province." [Online]. Available: https://www.sohu.com/a/369111094_99965141. 
[24] Y. Zhang, "Implementation of Word Frequency Analysis Platform and Algorithm Application," Comput. Program. Ski. Maint., pp. 109-111, 2019.

[25]L. Xin, "Feature selection based on word frequency difference and improved TF-IDF formula," $J$. Comput. Appl., vol. 25, pp. 2031-2033, 2005.

[26]Z. Bo, “MV's communication and value from musical society’s perspective," Nanjing, 2005.

[27] R. Lu, “Audience-oriented Strategy of Music Communication in the New Media era," North. Music, vol. 39, pp. 242-244, 2019.

[28] W. Shuang, "Song of the soul with free and easy freedom - talk about the song of original works of music of Chinese network," 2008.

[29]“Jin Opera.” [Online]. Available: https://baike.baidu.com/item/晋剧.

[30]“Shaanxi Opera.” [Online]. Available: https://baike.baidu.com/item/秦腔/596. 